\title{
Effects of Stray Capacitance to Ground in Three Electrode Monopolar Needle Bioimpedance measurements
}

\author{
H. Kalvøy, C. Aliau-Bonet Member, IEEE, R. Pallas-Areny Fellow, IEEE, Ø.G. Martinsen Senior \\ Member, IEEE
}

\begin{abstract}
Positive phase angle is documented and analyzed in a three electrode monopolar needle measurement. Inductance equivalent behavior of the stray capacitance to ground is described as error source in a non-inductive sample measurement.
\end{abstract}

\section{INTRODUCTION}

During development of new bioimpedance based applications (e.g. as described in Kalvøy [1]), we have had unexplained detections of positive phase angle in some of our measurements. These have been both for in-vitro laboratory setups and in clinical in-vivo experiments. Some of this behavior has been explained for 4-electrode setups by Grimnes and Martinsen [7]), but positive phase angle have also occurred in our monopolar 3-electrode Solartron ${ }^{\circledR}$ $1260 / 1294$ setups. This has typically been at frequencies between $100 \mathrm{kHz}$ and $1 \mathrm{MHz}$. The magnitude has been higher than expected from the inductive properties of tissue and it seems not to be fully explained by Grimnes and Martinsen, nor have we been able to avoid the positive phase by different optimizations of our 3-electrode setup. However, Aliau-Bonet and Pallas-Areny[4] addressed stray capacitance to ground as a contributor to apparent inductive behavior in 4-electrode impedance measurements. A manipulation of their 4-electrode equations showed that similar phenomenon may also occur in other setups, and in our hospital environment we have a lot of electronic equipment and large conductive surfaces representing possible stray capacitances between a human body surface and ground.

From this we postulate that stray capacitance can partly explain the positive phase angle in our 3-electrode Solartron ${ }^{\circledR} 1260 / 1294$ measurement setup. In this paper we have tested this hypothesis by evaluating the theory of AliauBonet and Pallas-Areny on an equivalent circuit modified for 3-electrode setups. Laboratory measurements were also performed to confirm the results from the theoretical analysis. The results have been used to propose strategies to avoid or minimize possible errors.

H. Kalvøy is with the Clinical and Biomedical engineering department at Rikshospitalet, Oslo University Hospital, Oslo, Norway. (corresponding author; phone: +47 2307 15; e-mail: havard.kalvoy@ous-hf.no).

C. Aliau-Bonet is with Universitat Politècnica de Catalunya, BarcelonaTech (UPC), Castelldefels, Spain, (e-mail: carles.aliau@upc.edu).

R. Pallas-Areny is with Universitat Politècnica de Catalunya, BarcelonaTech (UPC) Castelldefels, Spain (e-mail: ramon.pallas@upc.edu).

Ø.G. Martinsen is with the Department of Physics at the University of Oslo, Norway and the Clinical and Biomedical engineering department at Rikshospitalet, Oslo University Hospital, Oslo, Norway (e-mail: ogm@fys.uio).

\section{MATERIALS \& METHODS}

\section{A. Three electrodes bioimpedance measurement}

A large piece of bacon was used as sample. The type of biomaterial used is not critical for this study, and bacon has earlier been suggested as a convenient in vitro model for animal or human tissue [2]. A three electrode measurement was implemented by a 1294 impedance interface connected to a 1260 frequency response analyzer (Solartron Analytical [3]). The active measurement electrode $\left(I_{\text {in }}\right.$ in Fig 1$)$ was a non-insulated stainless steel needle cannula $(18 \mathrm{G} \times 50 \mathrm{~mm}$, Microlance 3, BD New Jersey, US) inserted $1 \mathrm{~cm}$ into a fatty part of the sample. The $V_{\mathrm{inL}}$ and $V_{\mathrm{inH}}$ electrodes were common ECG-electrodes (Blue sensor Q-00-A Ambu Medicotest, DK) placed on the skin of the sample. Signal ground of the transimpedance amplifier that measures current in the 1294 is "0 V floating" [3]. This electric potential reference node is not directly connected to ground (earth) potential but it is at the same potential. The sample under test was placed at a height $h$ with respect to the equipment (Fig. $1)$. The metal enclosure of the 1260 analyzer was earth grounded hence the height $(h)$ between the sample and the metal enclosure determined the stray capacitance between the sample and ground, which will increase for decreasing heights. Our aim is to reduce the effect of stray capacitances to ground in three electrode impedance measurements.

Fig. 1 is the equivalent circuit of the proposed setup, which includes the measurement functions of the 1294 interface and the sample with the positions of the electrodes. The voltage follower between the Solartron 1260 enclosure in Fig. 1 and signal ground describes the fact that signal ground is at earth-ground potential but not directly tied to it. If the stray capacitance between the sample and ground $\left(C_{\mathrm{g}}\right.$ in Fig. 1 ) is not negligible then current $I_{\mathrm{g}}$ through $C_{\mathrm{g}}$ will not be zero. $I_{\mathrm{g}}$ will not reach the current measurement electrode $\left(I_{\text {in }}\right)$ hence the impedance will be $Z=V_{\text {in }} / I_{\mathrm{L}}$, and since $I_{\mathrm{L}}=I_{\mathrm{H}}-I_{\mathrm{g}}$ (the amplifiers are assumed to be ideal), $Z$ will be larger than that when $I_{\mathrm{g}}=0$ (ideal measurement) $[3,4]$.

To minimize the effects of the stray capacitance to ground in the volume of the sample between low potential electrode $\left(V_{\text {inL }}\right)$ and measurement current electrode $\left(I_{\text {in }}\right)$ (Fig. 1), we place $V_{\text {inL }}$ and $I_{\text {in }}$ electrodes next to each other $(5 \mathrm{~cm}$ apart compared to the distance between $V_{\text {inH }}$ and $V_{\text {inL }}$ electrodes, 19 $\mathrm{cm}$ apart. Since $I_{\text {in }}$ is at ground potential, the density of electric field lines (displacement current) between the sample and the metal enclosure of the 1260 analyzer (ground plane) will be higher on the side at higher potential [6] (volume of the sample next to $V_{\text {inH }}$ electrode). Therefore, $C_{\mathrm{g}}$ is mainly due to the volume of the sample between the two potential electrodes ( $V_{\text {inH }}$ and $V_{\text {inL }}$ in Fig. 1). 


\section{RESULTS}

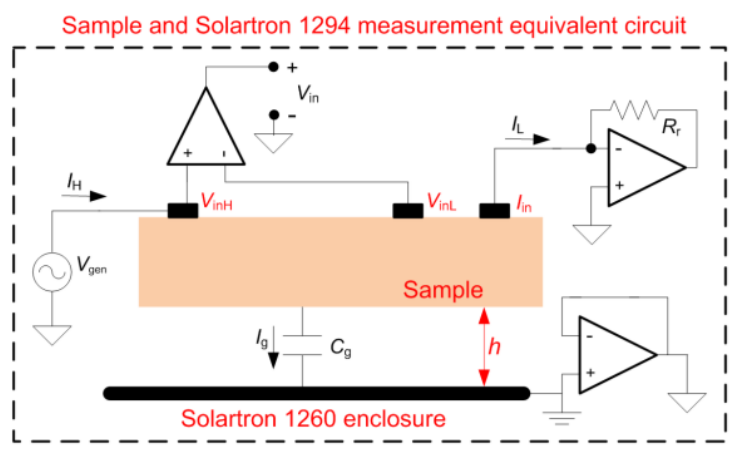

Figure 1. Equivalent circuit for the measurement setup. $V_{\mathrm{inH}}, V_{\mathrm{inL}}$ and $I_{\mathrm{in}}$ are electrodes (red color).

\section{B. Equivalent circuit}

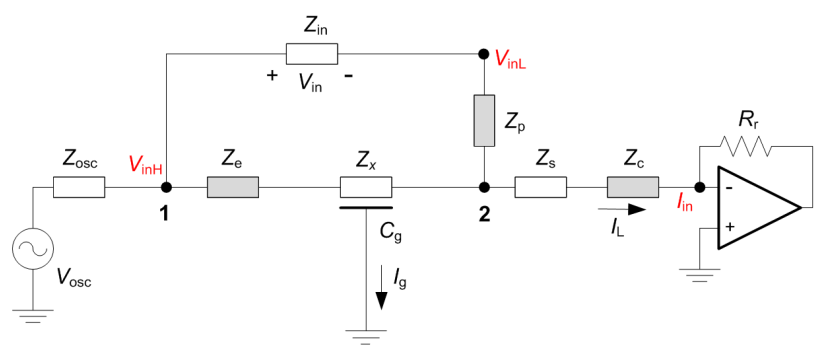

Figure 2. Circuit model where the stray capacitance from the sample to ground is a distributed element.

The equivalent circuit used as an electrical model for the measurement setup is shown in Fig. 2, which includes the equivalent discrete circuit component for each of the electrodes and the input impedance of the voltage amplifier used to measure the actual drop in voltage across the sample. $C_{\mathrm{g}}$ is shown as a distributed capacitance. Impedances with white color are volume impedances (conductors) and impedances with gray color are interface impedances (electrode impedances). $Z_{\mathrm{e}}$ is the impedance of the electrode where a sinusoidal voltage is applied to the sample and voltage is measured ( $V_{\mathrm{inH}}$, high potential measurement). $Z_{x}$ is the impedance of the sample and $Z_{\mathrm{s}}$ is the segmental impedance between low potential measurement electrode $\left(V_{\text {inL }}, Z_{\mathrm{p}}\right)$ and current measurement electrode $\left(I_{\text {in }}\right) . Z_{\text {in }}$ is the input impedance of the voltage amplifier.

In order to include the stray capacitance from the sample to ground in the lumped-parameter circuit model, we use the coefficient $\alpha$, which value is between 0 and 1 [4].

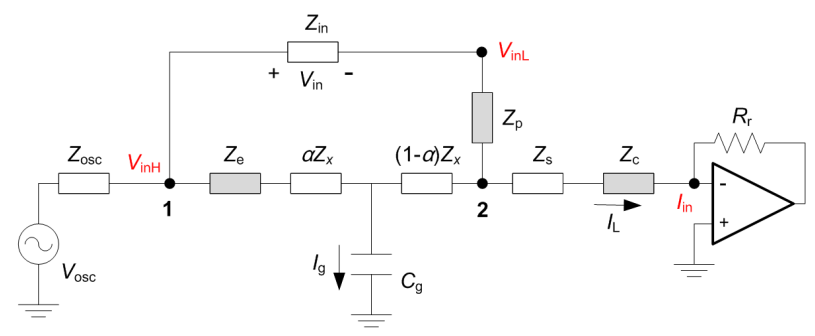

Figure 3. Circuit model where the stray capacitance from the sample to ground is a lumped element at an undetermined position.

\section{A. Equivalent circuit analysis}

To obtain the expression of the impedance measured $\left(V_{\text {in }} / I_{\mathrm{L}}\right)$ we have analyzed the circuit in Fig. 3. The detailed analysis is in the Appendix. The impedance measured is

$$
Z_{\mathrm{m}}=\frac{V_{\text {in }}}{I_{\mathrm{L}}}=\frac{Z_{\mathrm{in}}}{Z_{\mathrm{in}}+Z_{\mathrm{p}}+Z_{12}}\left\{Z_{\mathrm{c}}\left[1+\frac{\left(Z_{\mathrm{s}}+Z_{\mathrm{c}}\right)}{Z_{\mathrm{g}}}\right]+Z_{x}\left[1+(1-\alpha) \frac{Z_{\mathrm{e}}}{Z_{\mathrm{g}}}+\alpha \frac{\left(Z_{\mathrm{s}}+Z_{\mathrm{c}}\right)}{Z_{\mathrm{g}}}+\alpha(1-\alpha) \frac{Z_{\mathrm{s}}}{Z_{\mathrm{g}}}\right]\right\}
$$

If $Z_{\text {in }}$ is very large, we can approximate

$$
Z_{\mathrm{m}} \approx Z_{\mathrm{e}}\left[1+\frac{\left(Z_{\mathrm{s}}+Z_{\mathrm{c}}\right)}{Z_{\mathrm{g}}}\right]+Z_{x}\left[1+(1-\alpha) \frac{Z_{\mathrm{e}}}{Z_{\mathrm{g}}}+\alpha \frac{\left(Z_{\mathrm{s}}+Z_{\mathrm{c}}\right)}{Z_{\mathrm{g}}}+\alpha(1-\alpha) \frac{Z_{x}}{Z_{\mathrm{g}}}\right]
$$

where $Z_{\mathrm{g}}=\left(\mathrm{j} \omega C_{\mathrm{g}}\right)^{-1}$ and $Z_{12}$ is

$$
Z_{12}=Z_{\mathrm{e}}+Z_{x}\left[1+(1-\alpha) \frac{Z_{\mathrm{e}}}{Z_{\mathrm{g}}}+\alpha(1-\alpha) \frac{Z_{x}}{Z_{\mathrm{g}}}\right]
$$

The first term in the expression (1), is the voltage loading effect due to the fact that the input impedance of the voltage amplifier is not ideal (infinite). This effect depends on $C_{\mathrm{g}}$, which is included in $Z_{12}$, equation (2). This effect can be neglected as it is relevant at high frequency, above, say, 1 $\mathrm{MHz}$ [4], and the measurement frequency range of the 1294 is up to $0.75-1 \mathrm{MHz}$ [3]. Then, the expression of the measured impedance is the equation (1a), which also depends on $C_{\mathrm{g}}$. If $C_{\mathrm{g}}=0$, then $Z_{\mathrm{g}}$ is infinite and the measured impedance is $Z_{\mathrm{m}}=Z_{\mathrm{e}}+Z_{x}$, ideal result, But $C_{\mathrm{g}} \neq 0$ and the actual $Z_{\mathrm{m}}$ will be larger than $Z_{\mathrm{e}}+Z_{x}$ because both $Z_{\mathrm{e}}$ and $Z_{x}$ are multiplied by a factor larger than 1 (1 plus an error term, expressions inside the square brackets in (1b)). Therefore, $C_{\mathrm{g}}$ affects both impedances $Z_{\mathrm{e}}$ and $Z_{x}$. The term that affects $Z_{\mathrm{e}}$ depends on the segmental impedance $Z_{\mathrm{s}}$, the impedance $Z_{\mathrm{c}}$ of the current measurement electrode and the stray impedance to ground $Z_{\mathrm{g}}$. The terms that affect $Z_{x}$ depend on $\alpha$, the impedance $Z_{\mathrm{e}}$ of the injecting electrode, the impedance $Z_{\mathrm{c}}$ of the current measurement electrode, the segmental impedance $Z_{\mathrm{s}}$, the stray impedance to ground $Z_{\mathrm{g}}$ and itself $\left(Z_{x}\right.$, non-linear effect [4]). The effect of $C_{\mathrm{g}}$ on the measured impedance $Z_{\mathrm{m}}$ will depend on the kind of the impedances involved in the expression. We can generalize the terms in (1a) as

$$
Z_{k}\left[1+\sum_{i} c_{i} \frac{Z_{i}}{Z_{\mathrm{g}}}\right]=Z_{k}\left[1+\mathrm{j} \omega C_{\mathrm{g}} \sum_{i} c_{i} Z_{i}\right]
$$

$Z_{k}$ can be $Z_{\mathrm{e}}$ or $Z_{x}, Z_{i}$ can be $Z_{\mathrm{e}}, Z_{\mathrm{c}}, Z_{\mathrm{s}}$ and $Z_{x}$, and $c_{i}$ can be 1 , $\alpha,(1-\alpha)$ and $\alpha(1-\alpha)$. The coefficient $c_{i}$ is a real number and its effect is only an attenuation of the error term. If $Z_{i}=R_{i}$ is resistive, the error term $Z_{i} / Z_{\mathrm{g}}=\mathrm{j} \omega R_{i} C_{\mathrm{g}}$, this is an inductive effect, the impedance (magnitude and phase shift) increases when the frequency increases, and affect the impedance from $100 \mathrm{kHz}$ or even lower frequencies [4]. If $Z_{i}=\left(\mathrm{j} \omega C_{i}\right)^{-1}$ is capacitive, the error term $Z_{i} / Z_{\mathrm{g}}=C_{\mathrm{g}} / C_{i}$, this is a frequencyindependent error and affects the impedance in the overall measurement range [4]. When the ratio $C_{\mathrm{g}} / C_{\mathrm{i}}<<1$, there is not effect in the measured impedance [5].

If $Z_{i}$ has this form $\quad Z_{i}=R_{i}+\frac{R}{1+\mathrm{j} \omega R C_{i}}$

Then the expression of the error term will be

$$
\mathrm{j} \omega Z_{i} C_{\mathrm{g}}=\mathrm{j} \omega C_{\mathrm{g}}\left(R_{i}+\frac{R}{1+\left[\omega R C_{i}\right]^{2}}\right)+\frac{\left[\omega R C_{i}\right]^{2}}{1+\left[\omega R C_{i}\right]^{2}}\left(\frac{C_{\mathrm{g}}}{C_{i}}\right)
$$


The first term would be like an "inductive" effect and the second term would be like a "frequency-independent error". But, in both cases, the dependence on the frequency is more complex. If the $Z_{k}$ has a form similar to $Z_{i}$, then the equivalent circuit of the measured impedance could contain parallel resonant RLC circuits [5].

At high frequencies $(100 \mathrm{kHz}-1 \mathrm{MHz})$ the impedances are

$$
\begin{aligned}
& Z_{x}=R_{x}+\left.\frac{R_{x x}}{1+\mathrm{j} \omega R_{x x} C_{x}}\right|_{\omega R_{x x} C_{x}>>1} \approx R_{x} \\
& Z_{\mathrm{s}}=R_{\mathrm{s}}+\left.\frac{R_{\mathrm{ss}}}{1+\mathrm{j} \omega R_{\mathrm{ss}} C_{\mathrm{s}}}\right|_{\omega R_{\mathrm{ss}} C_{\mathrm{s}}>>1} \approx R_{\mathrm{s}} \\
& Z_{\mathrm{e}}=R_{\mathrm{e}}+\left.\frac{R_{\mathrm{ee}}}{1+\mathrm{j} \omega R_{\mathrm{ee}} C_{\mathrm{e}}}\right|_{\omega R_{\mathrm{ec}} C_{\mathrm{e}}>>1} \approx R_{\mathrm{e}} \\
& Z_{\mathrm{c}}=R_{\mathrm{c}}+\left.\frac{R_{\mathrm{cc}}}{1+\mathrm{j} \omega R_{\mathrm{cc}} C_{\mathrm{c}}}\right|_{\omega R_{\mathrm{cc}} C_{\mathrm{c}}>>1} \approx R_{\mathrm{c}}
\end{aligned}
$$

When $100 \mathrm{kHz}>>\left(2 \pi R_{i i} C_{i}\right)^{-1}$, where $i$ can be $\mathrm{x}, \mathrm{s}$, e and $\mathrm{c}$, then equation (1a) becomes

$$
\begin{aligned}
Z_{\mathrm{m}} & \approx R_{\mathrm{e}}\left[1+\mathrm{j} \omega C_{\mathrm{g}}\left(R_{\mathrm{s}}+R_{\mathrm{c}}\right)\right]+R_{x}\left[1+\mathrm{j} \omega C_{\mathrm{g}}\left\{(1-\alpha) R_{\mathrm{e}}+\alpha\left(R_{\mathrm{s}}+R_{\mathrm{c}}\right)+\alpha(1-\alpha) R_{x}\right\}\right] \\
& =R_{\mathrm{e}}+R_{x}+\mathrm{j} \omega C_{\mathrm{g}}\left[R_{\mathrm{c}}\left(R_{\mathrm{s}}+R_{\mathrm{c}}\right)+R_{x}\left\{(1-\alpha) R_{\mathrm{e}}+\alpha\left(R_{\mathrm{s}}+R_{\mathrm{c}}\right)+\alpha(1-\alpha) R_{x}\right\}\right] \\
& =R_{\mathrm{c}}+R_{x}+\mathrm{j} \omega L_{\mathrm{eq}} \\
L_{\mathrm{eq}} & =C_{\mathrm{g}}\left[R_{\mathrm{c}}\left(R_{\mathrm{s}}+R_{\mathrm{c}}\right)+R_{x}\left\{(1-\alpha) R_{\mathrm{c}}+\alpha\left(R_{\mathrm{s}}+R_{\mathrm{c}}\right)+\alpha(1-\alpha) R_{x}\right\}\right]
\end{aligned}
$$

Equation (10) corresponds to an equivalent circuit of the measured impedance like that in Fig. 5.

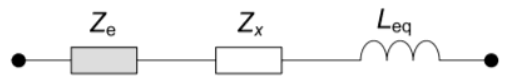

Figure 4. Equivalent circuit model of the measured impedance.

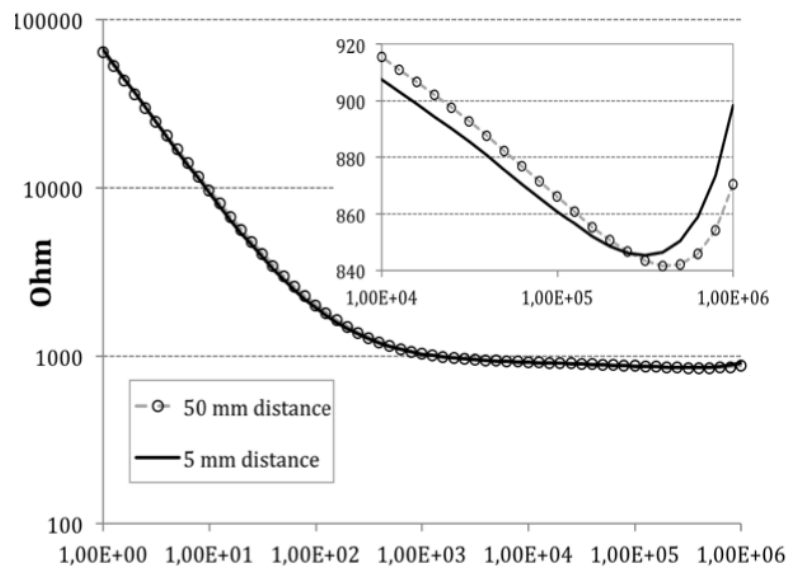

Figure 5 Dubble logarithmic plot of measured impedance modulus as function of frequency. Inset shows details from the highest frequencies on a stretched linear y-axis from 840 to $920 \mathrm{ohms}$.

\section{B. In-vitro measurements}

The measured impedance modulus (Fig. 5) and phase angle (Fig. 6) show a significant effect of electrode impedance for frequencies up to $1-10 \mathrm{kHz}$. This low frequency phase shift is related to the relatively small electrode area of the needle electrode $[1,7]$. No significant effects caused by the change on the $C_{\mathrm{g}}$ value are expected at low frequency.

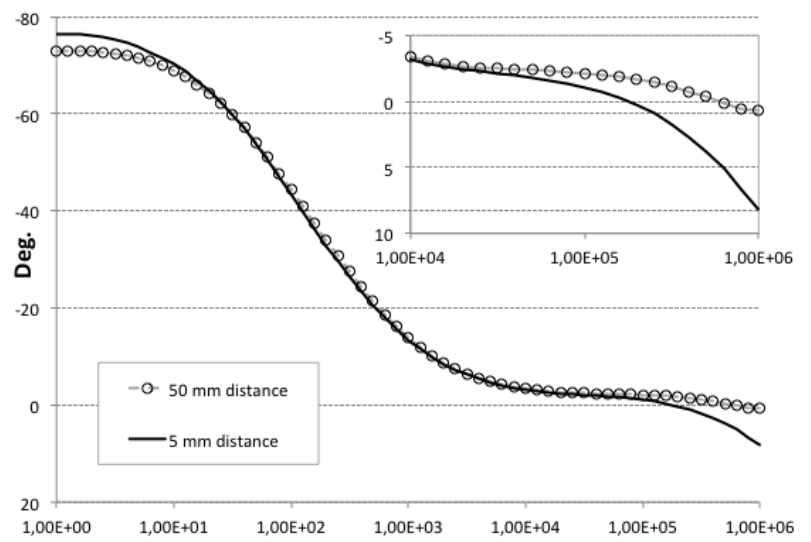

Figure 6 Measured phase angle as function of frequency (logarithmic $x-$ axis). Inset shows details from the highest frequencies on a stretched y-axis from -5 to 10 degrees (reversed dirrection).

The effects of $C_{\mathrm{g}}$ are visible at high frequency, between $100 \mathrm{kHz}$ and $1 \mathrm{MHz}$ in the modulus of impedance, where the modulus increases when the value of $C_{\mathrm{g}}$ increases. The effects on the phase shift of impedance are more visible, between $10 \mathrm{kHz}$ and $1 \mathrm{MHz}$, where the phase shift increases when the value of $C_{\mathrm{g}}$ increases. The difference, between phase shift at highest value of $C_{\mathrm{g}}$ and phase shift at lowest value of $C_{\mathrm{g}}$, increases with the frequency, inductive effect.

The modulus of impedance of the sample between $10 \mathrm{kHz}$ and $1 \mathrm{MHz}$ seems unaffected of electrode impedance, and is in accordance with previous publicized measurements in fat [1] except for the inductor-like increase above $200 \mathrm{kHz}$. If we model the almost flat level as a resistor, then $Z_{k}$ will be a resistance. $Z_{i}$ is also a resistance (in the range from $10 \mathrm{kHz}$ to $1 \mathrm{MHz}$ ) because no frequency dependent error is displayed in the measurements, then the ratio $C_{\mathrm{g}} / C_{i}<<1$.

\section{DISCUSSIONS}

A significant positive phase angle was found at the high end of the frequency spectrum measured in the sample for $h$ $=5 \mathrm{~mm}$ (inset in Fig. 6.). A large reduction of the positive phase angle was obtained by changing to $h=50 \mathrm{~mm}$ and keep all other factors unchanged. The analyses of the proposed equivalent circuit resulted in a theoretical equivalent inductance $\left(L_{\mathrm{eq}}\right)$ that represented the analytical link between the stray capacitance to ground and this measured positive phase phenomenon. Stray capacitance to ground is not easily shielded from a patient or a clinician with a shielding plate or similar (Figure 3-15. Guarding technique (1) Agilent "Handbook"[9]). Hence, other strategies must be found.

Aliau-Bonet and Pallas-Areny [4] shows that the effect of stray capacitance can be reduced by minimize the distance between the current carrying electrode and the pick-up electrode. Gersing [10] has the same conclusion and states that "the more the lengths of the possible lines of current differ, the more positive are the phase values". However, other considerations can lead to the opposite conclusion. Shiffman [11] have described adverse effects due to close 
distance between the current-carrying and the pick-up electrodes in measurement of body segments where homogenous current distribution through the whole segment is crucial for reproducible results. But this problem will vanish for truly monopolar needle measurements setups since the spatial sensitivity zone is highly focused on the needle tip [12]. The role of highly conductive surface shunt paths e.g. associated with excessively moist skin that is addressed by Grimnes and Martinsen [7] can still be a problem in a clinical setting. We suggests that 3-electrode needle measurements is done with the skin electrodes attached on a dry surface some centimeters apart.

A phase shift between 5 and $50 \mathrm{~mm}$ measurement is seen for the lowest frequencies in Fig. 6. This is not in accordance with the equations presented above, but previous studies on stainless steel needle electrodes show that some temporal drift in the electrode polarization properties can be expected [8]. However, this sub kilohertz-range drift will not preclude the results based on the frequencies above this range.

We did some simple test moving the sample about half a meter from the Solartron equipment and introducing stray capacitance by letting the needle operator hold his naked fingers on the needle during the measurement. The resultant phase angle was negative for the complete frequency spectra without operator contact, but during contact between the operator and the needle a positive phase angle occurred for the highest frequencies quite similar to what was found at $\mathrm{h}=$ $50 \mathrm{~mm}$ without contact (Fig 7). Further test with operators using typical clinical rubber gloves should be done to decide if continuous measurements during the needle insertion reliable, or recommendations for the clinicians to remove their hands and step away from the patient before each measurement should be give to avoid this error source.

\section{CONCLUSION}

Our bioimpedance measurements show that the hypothesized positive phase angle, caused by capacitive coupling to ground, is a possible source of error in a Solartron monopolar 3-electrode measurement setup. Analysis of the proposed stray capacitance equivalent circuit furthermore shows that this behavior is consistent with general circuit theory.

\section{APPENDIX}

To find $Z_{\mathrm{m}}=\mathrm{V}_{\mathrm{in}} / \mathrm{I}_{\mathrm{L}}$, expression (1) we can analyze the circuit of Fig. A1 (from Fig. 4), where $Z_{1}=Z_{e}+\alpha Z_{x}, Z_{2}=(1$ $-\alpha) Z_{x}$ and $Z_{3}=Z_{s}+Z_{c}$. The current and voltage equations of the circuit (Fig. A1) are

$$
\begin{aligned}
& I_{1}=I_{\mathrm{g}}+I_{2} \\
& I_{\mathrm{L}}=I_{\mathrm{in}}+I_{2} \\
& V_{12}=I_{\mathrm{in}}\left(Z_{\mathrm{in}}+Z_{\mathrm{p}}\right) \\
& V_{12}=I_{1} Z_{1}+I_{2} Z_{2} \\
& I_{\mathrm{g}} Z_{\mathrm{g}}=I_{2} Z_{2}+I_{\mathrm{L}} Z_{3} \rightarrow I_{\mathrm{g}}=I_{2} \frac{Z_{2}}{Z_{\mathrm{g}}}+I_{\mathrm{L}} \frac{Z_{3}}{Z_{\mathrm{g}}}
\end{aligned}
$$

From (A.5), (A.1) and (A.4) we obtain

$$
V_{12}=\left(I_{\mathrm{g}}+I_{2}\right) Z_{1}+I_{2} Z_{2}=\left(I_{2} \frac{Z_{2}}{Z_{\mathrm{g}}}+I_{\mathrm{L}} \frac{Z_{3}}{Z_{\mathrm{g}}}+I_{2}\right) Z_{1}+I_{2} Z_{2}
$$

Using (A.2)

$$
\begin{gathered}
V_{12}=I_{2}\left(Z_{1}+Z_{2}+\frac{Z_{1} Z_{2}}{Z_{\mathrm{g}}}\right)+I_{\mathrm{L}} \frac{Z_{1} Z_{3}}{Z_{\mathrm{g}}}=\left(I_{\mathrm{L}}-I_{\mathrm{in}}\right)\left(Z_{1}+Z_{2}+\frac{Z_{1} Z_{2}}{Z_{\mathrm{g}}}\right)+I_{\mathrm{L}} \frac{Z_{1} Z_{3}}{Z_{\mathrm{g}}} \\
V_{12}+I_{\text {in }}\left(Z_{1}+Z_{2}+\frac{Z_{1} Z_{2}}{Z_{\mathrm{g}}}\right)=I_{\mathrm{L}}\left(Z_{1}+Z_{2}+\frac{Z_{1} Z_{2}}{Z_{\mathrm{g}}}+\frac{Z_{1} Z_{3}}{Z_{\mathrm{g}}}\right) \\
Z_{12}=Z_{1}+Z_{2}+\frac{Z_{1} Z_{2}}{Z_{\mathrm{g}}}
\end{gathered}
$$

Using (A.3) and $\mathrm{I}_{\mathrm{in}}=\mathrm{V}_{\mathrm{in}} / \mathrm{Z}_{\text {in }}$

$$
\begin{aligned}
& \frac{V_{\text {in }}}{Z_{\text {in }}}\left(Z_{\text {in }}+Z_{\mathrm{p}}+Z_{12}\right)=I_{\mathrm{L}}\left(Z_{1}+Z_{2}+\frac{Z_{1} Z_{2}}{Z_{\mathrm{g}}}+\frac{Z_{1} Z_{3}}{Z_{\mathrm{g}}}\right) \\
& Z_{\mathrm{m}}=\frac{V_{\text {in }}}{I_{\mathrm{L}}}=\frac{Z_{\text {in }}}{Z_{\text {in }}+Z_{\mathrm{p}}+Z_{12}}\left(Z_{1}+Z_{2}+\frac{Z_{1} Z_{2}}{Z_{\mathrm{g}}}+\frac{Z_{1} Z_{3}}{Z_{\mathrm{g}}}\right)
\end{aligned}
$$

Replacing the expressions of $Z_{1}, Z_{2}$ i $Z_{3}$ in (A.11) we get the expression (1).

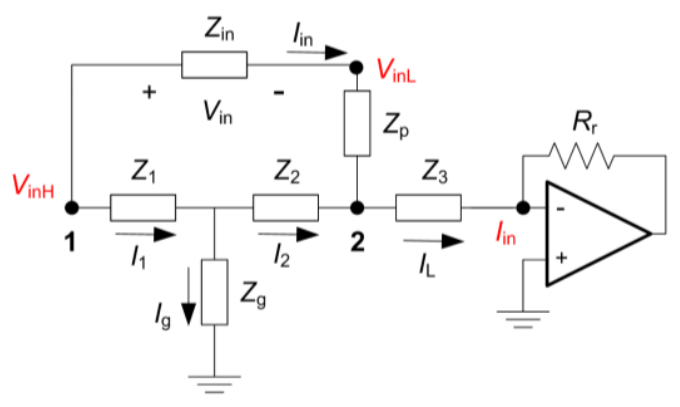

Figure A.1. Equivalent circuit

\section{REFERENCES}

[1] H. Kalvøy, L. Frich, S. Grimnes, Ø.G. Martinsen, P.K. Hol, A. Stubhaug, "Impedance-based tissue discrimination for needle guidance", Physiol. Meas. 30, 129-140, 2009

[2] H. Kalvøy, P. Høyum, S. Grimnes and Ø.G. Martinsen, "From impedance theory to needle electrode guidance in tissue" Journal of Physics: Conference Series 224(1), 2010

[3] Solartron 1294 "Operating Manual"

[4] C. Aliau-Bonet and R. Pallas-Areny, "On the effect of body capacitance to ground in tetrapolar bioimpedance measurements," IEEE Trans. Biomed. Eng., vol. 59, no. 12, pp. 3405-3411, Dec. 2012

[5] C. Aliau-Bonet and R. Pallas-Areny, "Effects of Stray Capacitance to Ground in Bipolar Material Impedance Measurements Based on Direct-Contact Electrodes," IEEE Trans. Instrum. Meas., 2014

[6] S. Ramo, J. R. Whinnery and T. Van Duzer, "Fields and waves in communication electronics", 3rd ed., New York: Wiley, 1994

[7] Grimnes, S. and Ø. G. Martinsen "Sources of error in tetrapolar impedance measurements on biomaterials and other ionic conductors." Journal of Physics D: Applied Physics 40(1): 9, 2007

[8] Kalvøy, H. Tronstad, C. Nordbotten, B. Grimnes, S. Martinsen, ØG. "Electrical Impedance of Stainless Steel Needle Electrodes", Annals of biomedical Engineering, 2010

[9] "The impedance measurement handbook, A guide to measurement technology and techniques," Agilent Technologies Co Ltd., 2009

[10] E. Gersing, H. Schafer, M. Osypka, "The appearance of positive phase angles in impedance measurements on extended biological objects" Innov. Tech. Biol. Med. 16 71-6, 1995

[11] C.A. Shiffman, "Adverse effects of near current-electrode placement in non-invasive bio-impedance measurements", Physiol. Meas. 34(11):1513-29, 2013

[12] P. Høyum, H. Kalvøy, Ø.G. Martinsen, S. Grimnes, "Finite element model of needle electrode spatial sensitivity", Physiol Meas. 31(10):1369-79, 2010 